\title{
A Low Voltage RF MEMS Variable Capacitor with Linear C-V Response
}

\author{
A. M. Elshurafa, P. H. Ho and K. N. Salama
}

An RF MEMS variable capacitor, fabricated in the PolyMUMPS process and tuned electrostatically, possessing a linear capacitance-voltage response is reported. The measured quality factor of the device was 17 at $1 \mathrm{GHz}$, while the tuning range was 1.2:1 and was achieved at an actuation DC voltage of $8 \mathrm{~V}$ only. Further, the linear regression coefficient was 0.98 . The variable capacitor was created such that it has both vertical and horizontal capacitances present. As the top suspended plate moves towards the bottom fixed plate, the vertical capacitance increases whereas the horizontal capacitance decreases simultaneously such that the sum of the two capacitances yields a linear capacitance-voltage relation.

Introduction: Most MEMS variable capacitors that are actuated electrostatically are of the parallel-plate type [1], and despite possessing high quality factors $(Q)$ they exhibit a nonlinear capacitance-voltage (CV) relationship because the tuning is taking place due to a transverse varying in the gap separating the two plates. Thus, research efforts have been directed to overcome this inherent nonlinearity. For example, the authors in [2] report a linear CV variable capacitor by developing a specific process whereby the suspended (movable) plate moves away from the other fixed plate. Moreover, the authors in [3] obtained a relatively linear response using a segmentedplate, but required a complicated fabrication process.

Contrary to conventional MEMS variable capacitors (or varactors), which only possess a vertical varying capacitance, the MEMS varactor proposed herein possesses varying horizontal and vertical capacitances; both these types of capacitances combined create the total capacitance of the varactor. As the top suspended plate moves towards the bottom fixed plate, the vertical (transverse) 
capacitance increases whereas the horizontal (lateral) capacitance decreases in such a way that the total capacitance change yields a linear response. The varactor was fabricated in the standard PolyMUMPS process.

Varactor Structure and Principle of Operation: A scanning electron microscope (SEM) image of the proposed variable capacitor structure is provided in Fig. 1a, where the general structure of the capacitor is shown along with the measurement pads, anchors and suspension arms. As can be seen, the total varactor contains two varactors that are identical in shape, mirror-imaged, and sharing a single set of measurement pads. Two variable capacitors were required because the capacitance magnitudes initially acquired from a single varactor were small to be used in real applications. The suspension arms are $100 \mu \mathrm{m}$ long and $14 \mu \mathrm{m}$ wide whereas the top plate is nearly square in shape with a side length of $260 \mu \mathrm{m}$. There is a total of eight suspension arms that hold the top plates of the overall varactor. Fig. $1 \mathrm{~b}$, on the other hand, shows a close up of the variable capacitor where the vertical and horizontal capacitances are indicated. Notice also the presence of the dimples in the top suspended plate which act as stoppers to ensure that the top plate does not collapse onto the bottom plate and cause shorting.

The top plate (signal) was created using the Poly2 and METAL layers. As for the bottom plate (ground), it was created using the Poly0 layer only when the vertical capacitance is needed, and was created using the Poly2 and METAL layers anchored to the underlying Poly0 layer when the horizontal capacitance was needed. Throughout the structure, the lateral separation between the signal and ground plates was $2 \mu \mathrm{m}$.

Fig. $1 \mathrm{~b}$ shows the capacitor structure before actuation $(0 \mathrm{~V})$, i.e. the overall capacitance of the variable capacitor is minimum. In this state, the vertical (transverse) capacitance is minimum because the top plate is furthest from the 
bottom plate. Conversely, the horizontal (lateral) capacitance is maximum because the suspended plate and the fixed plate are at the same altitude.

When the actuation voltage is applied, the suspended plate starts to move closer to the bottom fixed plate until the maximum overall capacitance is reached, which takes place when the dimples come in contact with the nitride. When this latter state is reached, the vertical capacitance is maximum because the suspended plate is as close as possible from the bottom plate, whereas the horizontal capacitance becomes minimum because the sides that used to be laterally facing each other are no longer so. Because the increase in the vertical capacitance is nonlinear, the horizontal capacitance decrease reduces the severity of the nonlinearity and yields linear overall change in capacitance.

Measurements: The tuning characteristics of the varactor at $1 \mathrm{GHz}$ are shown in Fig. 2 after de-embedding the capacitance of the pads. The minimum and maximum capacitances are $0.75 \mathrm{pF}$ and $0.9 \mathrm{pF}$ respectively, which translate into a tuning range of 1.2:1. On the same figure, a trend-line is plotted and shows a linear regression coefficient $\left(R^{2}\right)$ of 0.985 . The pull-in [4] takes place at $8.5 \mathrm{~V}$ and the capacitance then immediately rises to $2.6 \mathrm{pF}$.

The $Q$ at $1 \mathrm{GHz}$ was 17 and drops to 6 at $4 \mathrm{GHz}$ as shown in the sample measurement in Fig. 3. Further, at pull-in, $Q$ drops also to 6.2 at $1 \mathrm{GHz}$. However, throughout the tunable linear range, $Q$ was over 15 at $1 \mathrm{GHz}$.

We summarize the performance of the proposed varactor with other reported varactors in Table $1[2-3,5]$. The reported varactors in [2-3] obtain a higher tuning range when compared to the proposed varactor at the expense of a much higher required voltage and a specifically developed process. Further, the proposed varactor outperforms the one reported in [5] on all fronts. We also note that the reported $Q$ herein is higher than all previously reported.

Conclusion: A variable capacitor with a linear CV response fabricated using the standard PolyMUMPS process has been presented. Among the desirable 
characteristics of the proposed varactor is that it required a low voltage to achieve the actuation. Unlike previously reported variable capacitors which possessed vertical capacitances only, the proposed varactor relies on vertical and horizontal capacitance changes within its structure to achieve the linear CV relation. Future work includes further optimization of the device to attain a higher tuning range and higher capacitance values.

\section{References}

1 Dec A. and Suyama K.: 'Micromachined Varactor with Wide Tuning Range', Electronics Letters, 1997, 33, (11), pp. 922-924.

2 Han C. H., Choi D. H., Choi S. J. and Yoon J. B.: 'MEMS variable capacitor with superior linearity and large tuning ratio by moving the plate to the increasing-gap direction', IEEE MEMS Tech. Dig., 2011, pp. 772-77.

3 Shavezipur M., Khajepour A. and Hashemi S. M.: 'Development of Novel Segmented-Plate Linearly Tunable MEMS Capacitors', J. Micromech. Microeng., 2008, 18, (3), 035035.

4 Elshurafa A. M. and El-Masry E. I.: 'MEMS Variable Capacitance Devices Utilizing the Substrate: I. Novel Devices with Customizable Tuning Range', J. Micromech. Microeng., 2010, 20, (4), 045027.

5 Seohno S., Choi W. and Chun K.: 'A Novel Linearly Tunable MEMS Variable Capacitor', J. Micromech. Microeng., 2002, 12, (1), 8pp.

\section{Authors' affiliations}

A. M. Elshurafa, P. H. Ho and K. N. Salama are with King Abdullah University of Science and Technology (Electrical Engineering, Physical Sciences and Engineering Division, Thuwal 23955-6900, Saudi Arabia).

E-mail: elshurafa@ieee.org 


\section{Figure captions}

Fig. 1 A scanning electron microscope image of the fabricated variable capacitor in the PolyMUMPS process.

a The complete variable capacitor structure showing the measurement pads, anchors, and suspension arms.

b A close up SEM of the lower right corner highlighted in a dotted box in Fig. 1a, showing the top plate (Poly2+METAL), the bottom plate (Poly0), horizontal capacitances, vertical capacitances, and the dimple.

Fig. 2 Tuning characteristics of the proposed variable capacitor from $0 \mathrm{~V}$ up to $8 \mathrm{~V}$ (i.e. just before pull-in occurs at $8.5 \mathrm{~V}$ ). $R^{2}$ is the linear regression coefficient.

Fig. 3 A sample measurement for the varactor showing that $Q$ is 17 at $1 \mathrm{GHz}$ and 6 at $4 \mathrm{GHz}$.

Table Caption

Table 1 Comparative Study 
Figure 1a

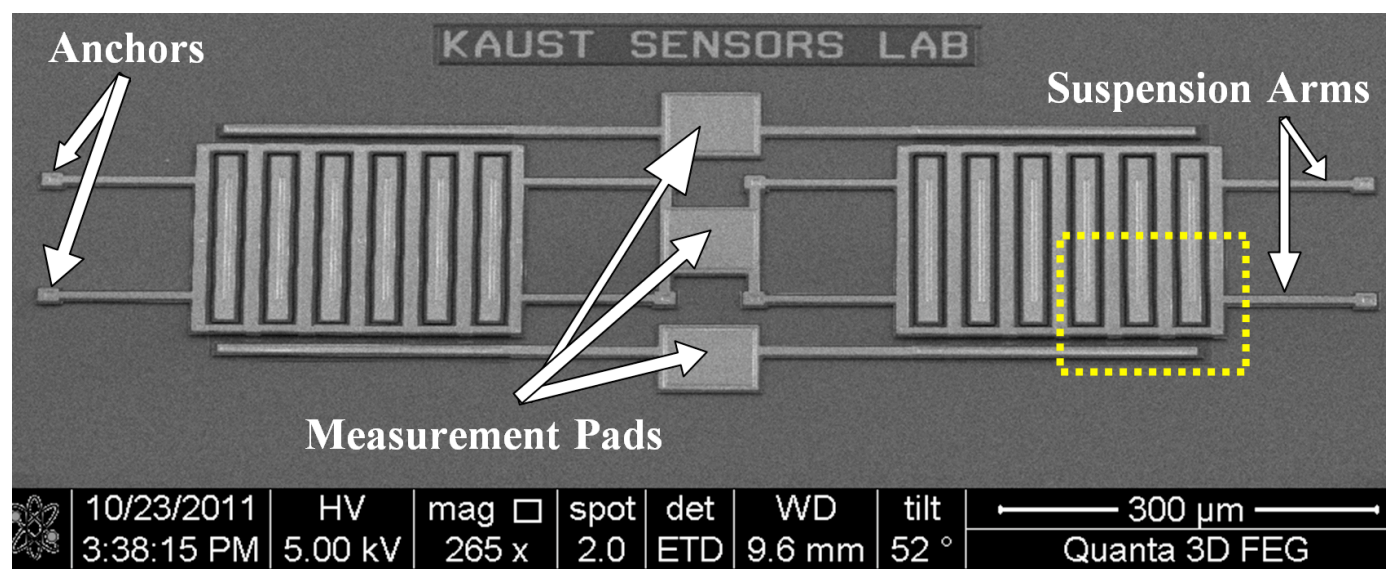


Figure 1b

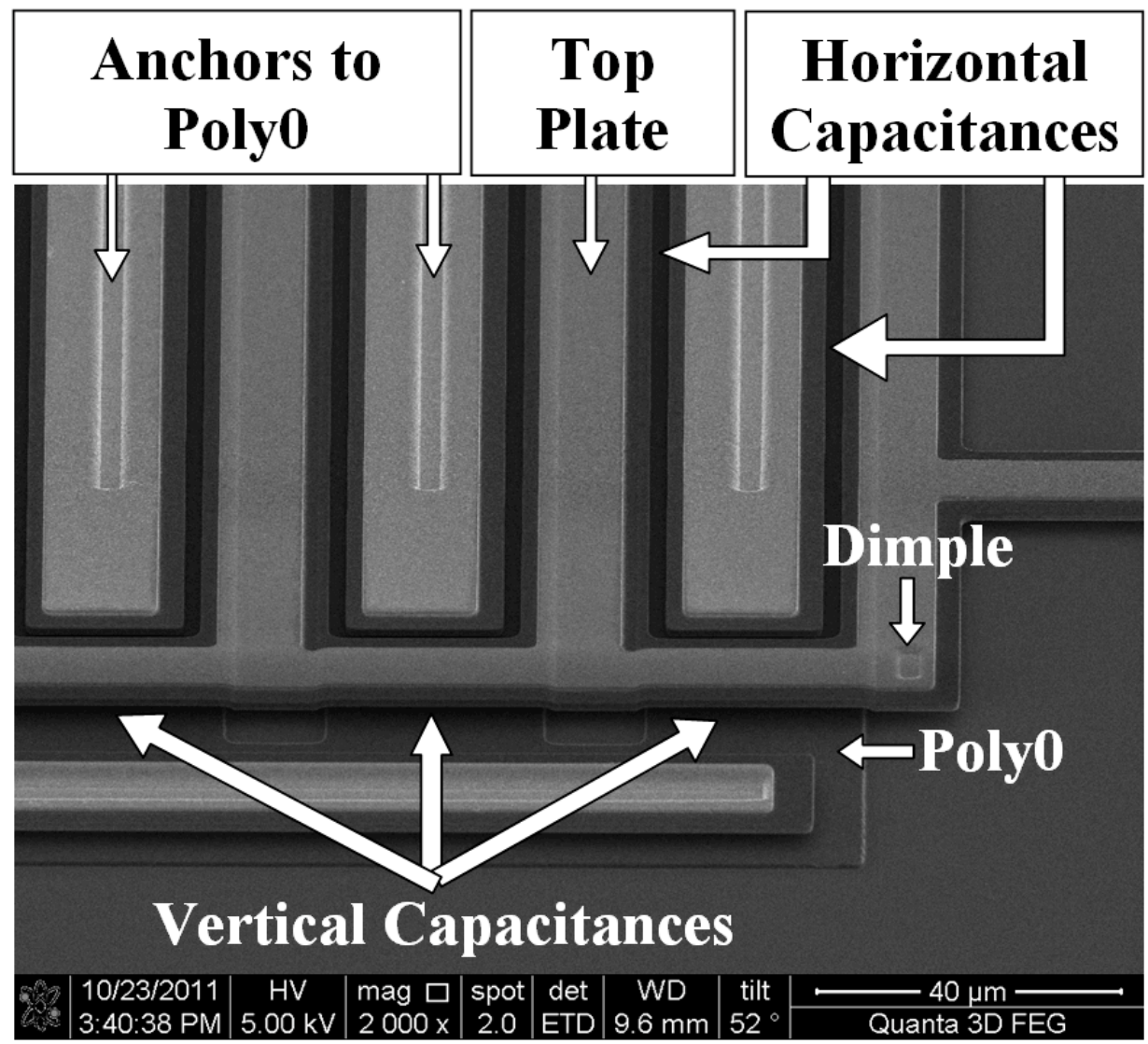

b 
Figure 2

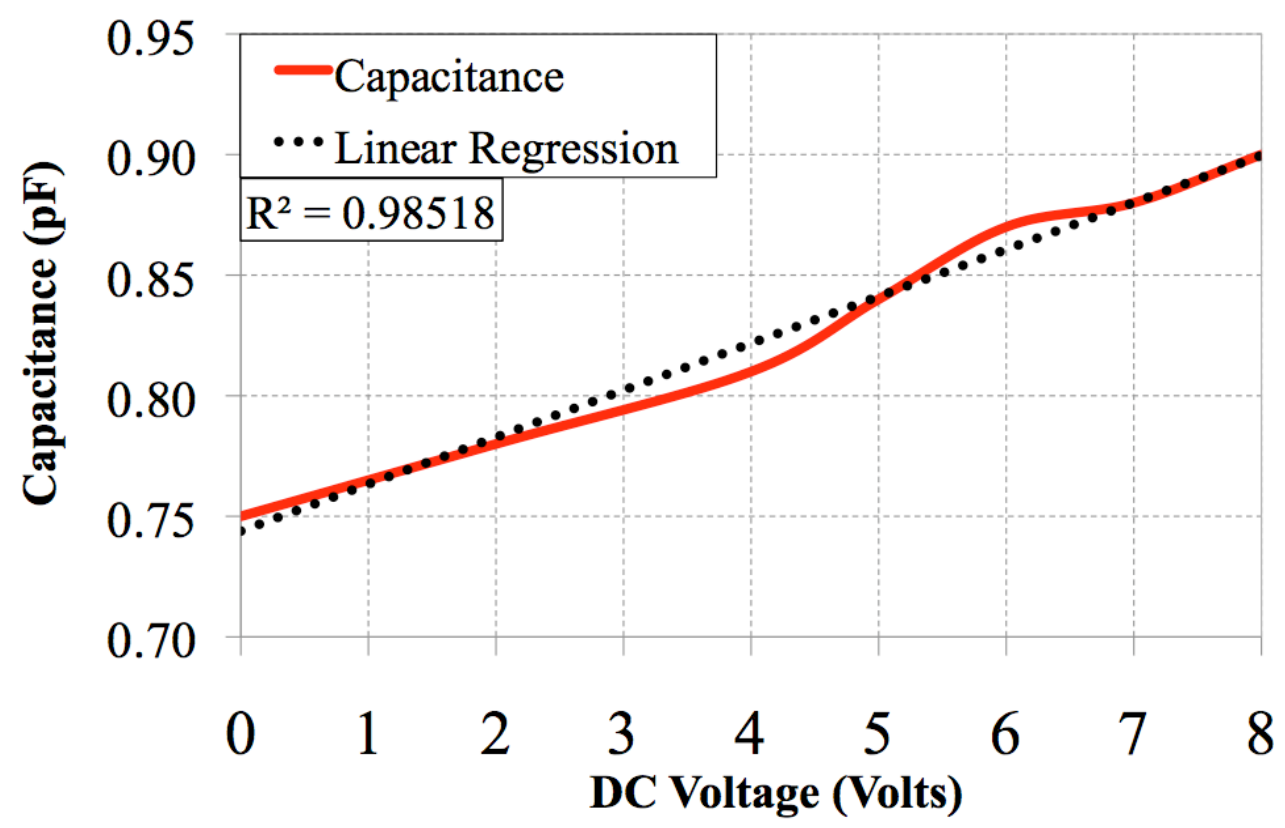


Figure 3

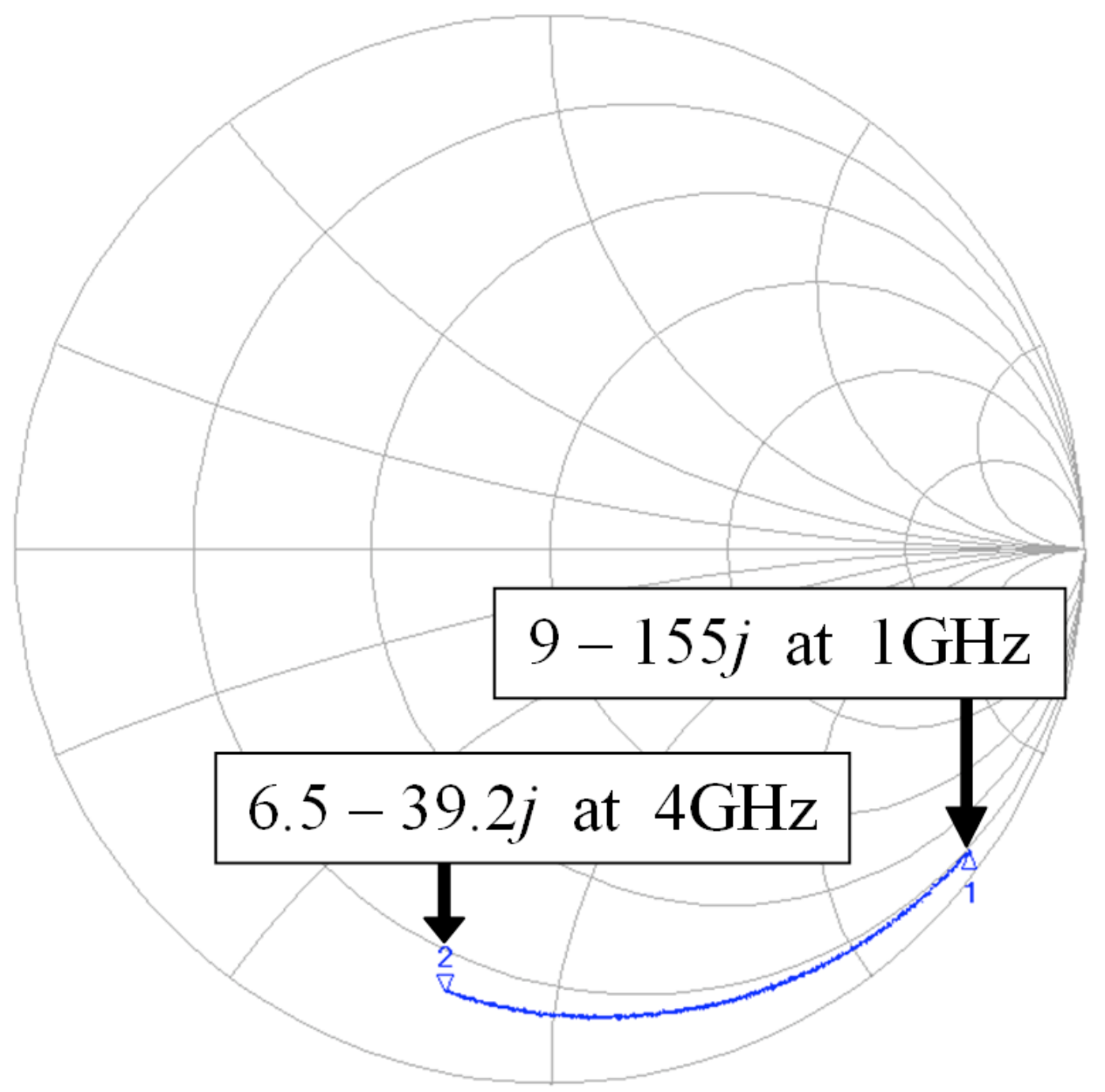


Table 1

\begin{tabular}{|c|c|c|c|c|c|}
\hline Ref. & $\begin{array}{l}\text { Tuning } \\
\text { Range }\end{array}$ & $\begin{array}{c}\text { Voltage } \\
\text { Requirement }\end{array}$ & $\begin{array}{l}\text { Process or } \\
\text { Technology }\end{array}$ & Linearity & $Q$ at $1 \mathrm{GHz}$ \\
\hline [2] & $200 \%$ & $45 \mathrm{~V}$ & $\begin{array}{l}\text { Specifically } \\
\text { developed }\end{array}$ & $R^{2}=0.984$ & 8 \\
\hline [3] & $\begin{array}{c}50 \% \\
\text { (Simulation) }\end{array}$ & $\begin{array}{c}50 \mathrm{~V} \\
\text { (Simulation) }\end{array}$ & $\begin{array}{c}\text { Specifically } \\
\text { developed; } \\
\text { bad } \\
\text { repeatability }\end{array}$ & N/A & $\mathrm{N} / \mathrm{A}$ \\
\hline [5] & $10 \%$ & $8 \mathrm{~V}$ & $\begin{array}{l}\text { Specifically } \\
\text { developed }\end{array}$ & N/A & 4 \\
\hline This work & $20 \%$ & $8 \mathrm{~V}$ & Standard & $R^{2}=0.985$ & 17 \\
\hline
\end{tabular}

